\title{
A young woman with abrupt changes in skin and mucosa
}

\author{
Vitorino Modesto dos Santos \\ Department of Internal Medicine, Armed Forces Hospital and Catholic University of Brasília, Brasília-DF, Brazil
}

\begin{abstract}
Cutaneous and mucosal changes due to adverse drug effects are relatively common in clinical daily practice; however, the etiology often follows unsuspected, undetermined and underreported. More severe lesions like the Stevens-Johnson syndrome should be early recognized and treated. As the entity is potentially lethal if not properly managed, and diagnosis is imminently clinical, the characteristic images might contribute to enhance the suspicion index about this syndrome.
\end{abstract}

\section{A young woman with abrupt changes in skin and mucosa}

A 36-year-old Brazilian woman was referred to hospital because of acute onset of skin erythema and purpuric macules, in association with oral, genital, and ocular erosions and bullae, which rapidly involved approximately $10 \%$ of the body surface. Her medical antecedents were unremarkable, except for the use of anti-inflammatory pills to control a tooth ache ten days ago. Physical examination showed an acutely ill female, hypotensive, with tachycardia and tachypnea but without fever. Major changes were observed in buccal, perioral, ocular, and genital areas; moreover, skin le-

Correspondence: Vitorino Modesto dos Santos, Armed Forces Hospital, Estrada do Contorno do Bosque s/n, Cruzeiro Novo, CEP 70.658-900, Brasília-DF, Brazil.

Tel.: +55.61.39662103 - Fax: +55.61.32331599.

E-mail: vitorinomodesto@gmail.com

Key words: Drug side-effect; skin rash; Stevens-Johnson syndrome.

Acknowledgments: the author would like to thank Almir José Batista, official photographer of the Armed Forces Hospital, Brasília-DF, Brazil for the images that illustrate the present manuscript.

Conflict of interest: the authors declare no potential conflict of interest.

Received for publication: 18 August 2016.

Revision received: 13 October 2016.

Accepted for publication: 17 November 2016.

This work is licensed under a Creative Commons Attribution NonCommercial 4.0 License (CC BY-NC 4.0).

CCopyright V. Modesto dos Santos, 2017

Licensee PAGEPress, Italy

Italian Journal of Medicine 2017; 11:340-342

doi:10.4081/itjm.2016.769 sions were detected on the extremities affecting palms and soles (Figure 1). Worthy of note, several skin lesions had the classical appearance of target, iris or bull's-eye. Laboratory tests revealed mild anemia, neutrophilic leukocytosis and elevated C-reactive protein. The results of microbiological studies, including cultures and serological analysis, were negative. Histopathological study of biopsy sample showed keratinocyte apoptosis, vacuolar changes, sub epidermal blisters, and dermal perivascular lymphocytic infiltrate permeated with eosinophils. The patient was clinically managed in the Intensive Care Unit and her improvement was slow. Her treatment included nutritional support, antibiotics, corticosteroids, and topical care of lesions. The cutaneous and mucosal changes gradually improved and were all healed in about 30 days. After the hospital discharge she remained asymptomatic and without recurrences of the lesions.

What is your diagnosis?

\section{Drug-induced Stevens-Johnson syndrome}

The patient herein described had clinical, laboratory and histopathological features consistent with the Stevens-Johnson syndrome (SJS), ${ }^{1-5}$ first described in 1922 in two patients evaluated in the tenth and the twenty-second days after the onset of illness. ${ }^{1,4}$ Their eyes were exuding purulent secretion, the lips had clotted blood, and the eruption affected almost all body except for the feet and hands, ${ }^{1}$ different from the present case. The blisters and desquamation in SJS classically involve mucosae and up to $10 \%$ of the body surface..$^{2-5}$ Toxic epidermal necrolysis (TEN) is a similar entity, which can be differentiated from SJS with base on the extension of over $30 \%$ of body surface area affected; whereas the overlap SJS/TEN is characterized by 10 to $30 \%$ of body surface involvement. ${ }^{2,4,5}$ Major causes of SJS are drug reactions and infections caused by Mycoplasma pneumoniae, Chlamydia pneumoniae, cytomegalovirus, human immunodeficiency virus, and disseminated candidia- 


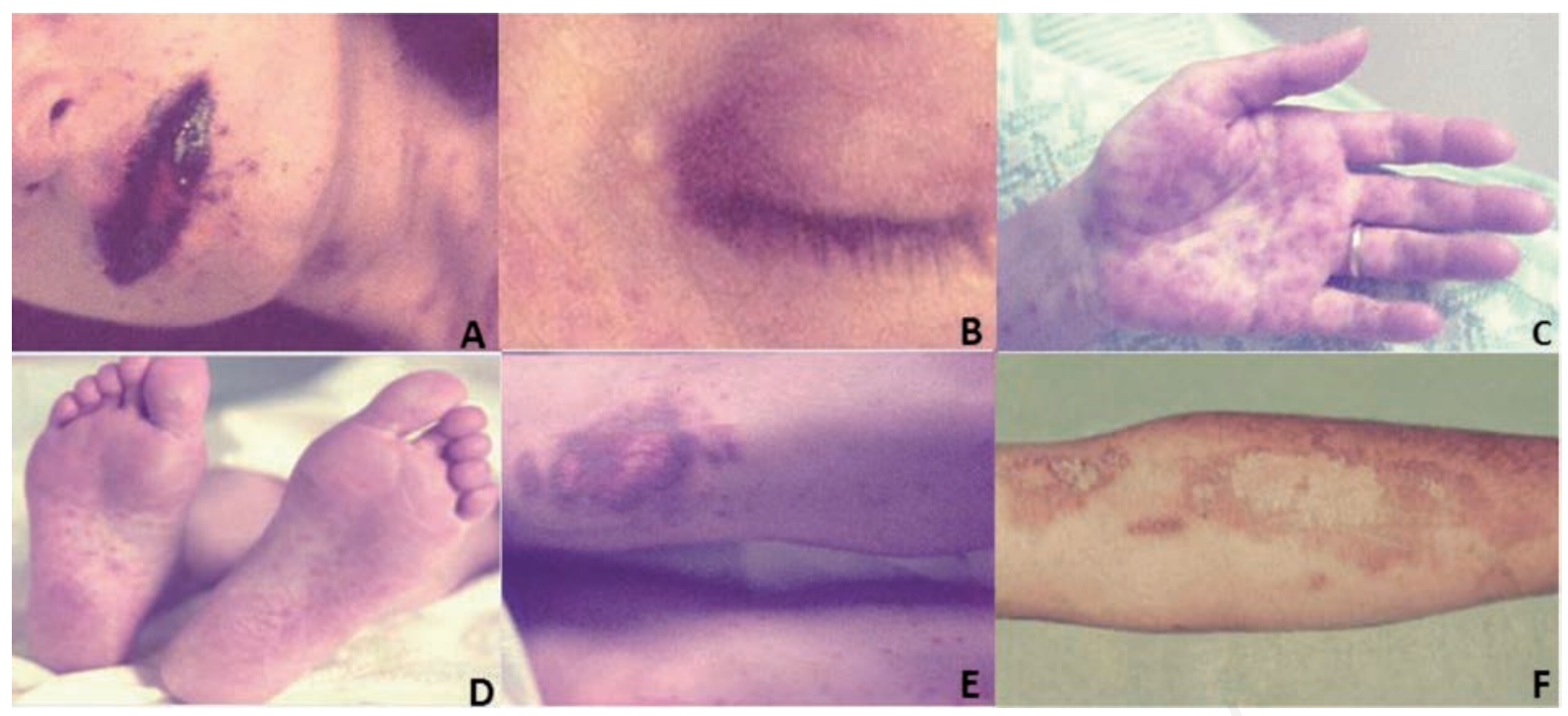

Figure 1. A-E) Presentation of the changes on admission showing hemorrhagic erosion and encrusted lesions on lips, ocular erythema, purpuric macules, multiple papules and erythematous rashes over extremities, involving palms and soles; and F) desquamative features of superficial healed lesions of the upper extremity.

sis. ${ }^{2,4}$ Management of patients with SJS includes prevention of secondary infection, fluid and electrolyte balance, nutrition support, glucocorticoids, antibiotics, and immunoglobulins. ${ }^{2,4}$ Systemic complications include respiratory, cardiovascular, gastrointestinal and renal involvement, ${ }^{4,5}$ and mortality rate may be up to $25 \% .{ }^{2}$ Chantaphakul et al. reviewed 24 patients with SJS in Thailand during a 5 -year period; $54.2 \%$ were males, with mean age 46.5 (20-77) years, and $33.3 \%$ with HIV infection. Oral, ocular, genital, and hepatic changes were observed in $100 \%, 83.3 \%, 41.7 \%$ and $41.7 \%$ of cases, respectively. ${ }^{2}$ Most of severe forms were related to adverse drug effects; $65 \%$ of cases were treated with systemic steroids during 1 to 10 days, and there was no mortality in this group of patients. ${ }^{2}$ Diphoorn et al. reviewed the incidence, drug exposure and mortality data of 59 cases of SJS in the Italian Lombardy region over a period of 5 years and a half. The majority of cases were associated with adverse drug reactions, mainly to allopurinol, and the mortality rate was $16.9 \%{ }^{3}$ Martinez-Cabriales et al. reviewed advances in clinical, pathogenesis and management of SJS and reported an annual incidence of 1.2 to 6.0 cases per million inhabitants, the growing incidence with age, and ethnic genetic predisposition. ${ }^{4}$ They commented the role of medications and infections by mycoplasma, cytomegalovirus and Dengue virus; and emphasized the need of histopathological confirmation, additionally to clinical features. ${ }^{4}$ Yamane et al. reviewed 52 cases of SJS during 2000-2013 in Japan. The mean age of patients was 55.1 years, and 31 were females; 53.85 of cases were due to adverse drug ef- fects. ${ }^{5}$ The average interval between the first causative drug intake and the onset of symptoms of SJS was 18 days, and main complications were pneumonia and sepsis. ${ }^{5}$ Before 2007 the mortality rate was $1.9 \%$, and decreased from $4.5 \%$ to $0.0 \%$ between 2007 and 2013 after addition of immunoglobulins and plasmapheresis to corticotherapy. ${ }^{5}$ Qayoom et al. studied adverse cutaneous drug reactions in the Kashmir valley in India during one year. The incidence was $0.16 \%$, the mean age of patients was $39.36 \pm 16.77$ years with a range of $2-75$ years; $50.66 \%$ were females, and the main route was oral $(86.66 \%) .{ }^{6}$ Fixed drug eruptions occurred in $45.33 \%$ and SJS in only $5.33 \%$ of the patients. Antimicrobials (57.33\%), nonsteroidal anti-inflammatory drugs $(21.33 \%)$, and antiepileptics $(17.33 \%)$ were the commonest. ${ }^{6}$

The present clinical images aim to enhance the suspicion index of primary care workers about severe drug-induced cutaneous reactions, and contribute to establish the earliest diagnosis.

\section{References}

1. Stevens AM, Johnson FC. A new eruptive fever associated with stomatitis and ophthalmia report of two cases in children. Am J Dis Child 1922;24:526-33.

2. Chantaphakul H, Sanon T, Klaewsongkram J. Clinical characteristics and treatment outcome of Stevens-Johnson syndrome and toxic epidermal necrolysis. Rev Surg 1966;23:1-4

3. Diphoorn J, Cazzaniga S, Gamba C, et al. Incidence, causative factors and mortality rates of Stevens-Johnson syndrome (SJS) and toxic epidermal necrolysis (TEN) 
in northern Italy: data from the REACT registry. Pharmacoepidemiol Drug Saf 2016;25:196-203.

4. Martínez-Cabriales SA, Gómez-Flores M, Ocampo-Candiani J. News in severe clinical adverse drug reactions: Stevens-Johnson syndrome (SJS) and toxic epidermal necrolysis (TEN). Gac Med Mex 2015;151:721-31.

5. Yamane Y, Matsukura S, Watanabe Y, et al. Retrospec- tive analysis of Stevens-Johnson syndrome and toxic epidermal necrolysis in 87 Japanese patients - Treatment and outcome. Allergol Int 2016;65:74-81.

6. Qayoom S, Bisati S, Manzoor S, et al. Adverse cutaneous drug reactions - a clinic-demographic study in a tertiary care teaching hospital of the Kashmir Valley, India. Arch Iran Med 2015;18:228-33. 